\title{
Capacity for diagnosis and treatment of heart failure in sub-Saharan Africa
}

\author{
Selma Carlson, ${ }^{1}$ Herbert C Duber ${ }^{2,3}$ Jane Achan, ${ }^{4}$ Gloria Ikilezi, ${ }^{3,4}$ Ali H Mokdad ${ }^{2}$ \\ Andy Stergachis, ${ }^{5,6}$ Alexandra Wollum, ${ }^{2}$ Gene Bukhman, ${ }^{7,8}$ Gregory A Roth ${ }^{1,2}$
}

'Division of Cardiology, University of Washington, Seattle, Washington, USA ${ }^{2}$ Institute for Health Metrics and Evaluation, Seattle, Washington, USA

${ }^{3}$ Division of Emergency Medicine, University of Washington, Seattle, Washington, USA

${ }^{4}$ Medical Research Council Unit, The Gambia, Banjul, Gambia ${ }^{5}$ Department of Pharmacy, University of Washington, Seattle, Washington, USA

Department of Global Health, University of Washington, Seattle, Washington, USA ${ }^{7}$ Program in Global NCDs and Social Change, Department of Global Health and Social Medicine, Harvard Medical School, Boston, Massachusetts, USA

${ }^{8}$ Cardiovascular Division and Division of Global Health Equity, Brigham and Women's Hospital, Boston, Massachusetts, USA

\section{Correspondence to}

Dr Selma Carlson, Division of Cardiology, University of Washington, 1959 Pacific St NE, Box 356422, Seattle WA 98195, USA; selmad@uw.edu

Received 15 November 2016 Revised 21 March 2017 Accepted 26 March 2017 Published Online First 10 May 2017

\section{Linked}

- http://dx.doi.org/10.1136/ heartjnl-2017-311558

\section{CrossMark}

To cite: Carlson S, Duber HC, Achan J, et al. Heart

2017:103:1874-1879.

\section{ABSTRACT \\ Objective Heart failure is a major cause of disease} burden in sub-Saharan Africa (SSA). There is an urgent need for better strategies for heart failure management in this region. However, there is little information on the capacity to diagnose and treat heart failure in SSA. We aim to provide a better understanding of the capacity to diagnose and treat heart failure in Kenya and Uganda to inform policy planning and interventions.

Methods We analysed data from a nationally representative survey of health facilities in Kenya and Uganda (197 health facilities in Uganda and 143 in Kenya). We report on the availability of cardiac diagnostic technologies and select medications for heart failure ( $\beta$-blockers, ACE inhibitors and furosemide). Facilitylevel data were analysed by country and platform type (hospital vs ambulatory facilities).

Results Functional and staffed radiography, ultrasound and ECG were available in less than half of hospitals in Kenya and Uganda combined. Of the hospitals surveyed, $49 \%$ of Kenyan and $77 \%$ of Ugandan hospitals reported availability of the heart failure medication package. ACE inhibitors were only available in $51 \%$ of Kenyan and $79 \%$ of Ugandan hospitals. Almost one-third of the hospitals in each country had a stock-out of at least one of the medication classes in the prior quarter.

Conclusions Few facilities in Kenya and Uganda were prepared to diagnose and manage heart failure. Medication shortages and stock-outs were common. Our findings call for increased investment in cardiac care to reduce the growing burden of heart failure.

\section{INTRODUCTION}

The burden of heart failure (HF) is of increasing concern as a major cause of cardiovascular disease in sub-Saharan Africa (SSA). ${ }^{1-4} \mathrm{HF}$ appears to occur at an earlier age and carry a higher rate of in-hospital mortality in SSA compared with other regions of the world. ${ }^{2}$ Non-ischaemic causes of HF, including hypertensive heart disease, rheumatic heart disease and non-ischaemic cardiomyopathy, appear to be particularly common. ${ }^{35}$

The WHO's Global Action Plan has put forth a target of a $25 \%$ relative reduction in overall mortality from cardiovascular diseases by $2025 .^{6}$ To accomplish this, the European Society of Cardiology has launched the Global Heart Failure Awareness Programme that outlines a comprehensive approach which includes the application of best practices in diagnosis, treatment and long-term management. ${ }^{7}$ For HF with reduced ejection fraction, this would include treatment with diuretics, ACE inhibitors and selected $\beta$-blockers. Guideline-directed medical therapy for HF has been shown to be cost-effective or even cost saving in low-income and middle-income countries (LMICs) such as those in SSA. ${ }^{89}$

The use of evidence-based medications for HF in SSA is poor, ${ }^{10}$ but access to and availability of these therapies is unknown. Using health facility data collected as part of the Access, Bottlenecks, Cost and Equity (ABCE) Study, we assessed the countrywide capacity to diagnose and treat HF in Kenya and Uganda. In particular, we examined the availability of standard diagnostic modalities and basic medications, including selected $\beta$-blockers, selected ACE inhibitors and a diuretic, for $\mathrm{HF}$ care.

\section{METHODS}

\section{Health facilities in Kenya and Uganda}

Kenya and Uganda have national health systems that are similarly organised into tiers (figure 1 ). The tiers are designed to deliver increasing levels of healthcare as it pertains to equipment and medication availability, infrastructure, medical expertise and acuity. National guidelines in both countries classify medications, equipment and supplies by the lowest tier of intended use (and are assumed to be available at higher tiers). Hospital tiers differ slightly in title between the two countries. Subdistrict hospitals in Kenya and health centre IVs in Uganda represent analogous healthcare tiers and are the tier at which most HF medications and diagnostic equipment are expected to be available. Among the different ambulatory platforms, clinics represent the lowest tier in both countries and provide basic curative services. They are typically managed by one health professional. In Kenya, other ambulatory facilities include maternity homes and health centres, the latter of which serve as the country's main primary care facilities. In Uganda, other ambulatory facilities include health centre IIs and health centre IIIs, which differ in that health centre IIIs serve a larger population and provide some basic inpatient and laboratory services.

\section{Survey design and data collection}

The ABCE study utilised health facility surveys to collect information on a nationally representative group of health facilities in Kenya and Uganda in 2011 and 2012. The methods of the ABCE study have been previously reported. ${ }^{11-13}$ In 


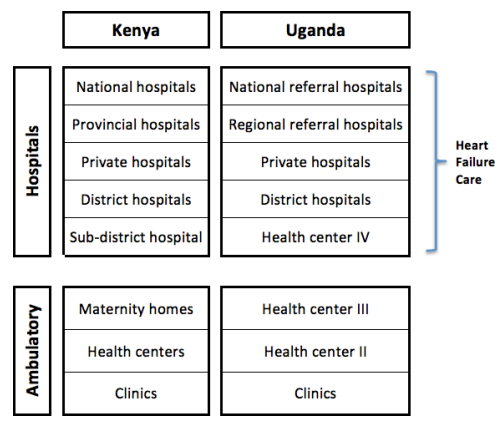

Figure 1 Overview of healthcare systems in Kenya and Uganda. The healthcare systems in Kenya and Uganda are similarly organised in tiers. HF care is provided at hospital level facilities (subdistrict hospitals and above in Kenya, health centre IVs and above in Uganda).

brief, health facility selection for ABCE used a two-step stratified random sampling process. In Kenya, counties were stratified into 27 groups based on rates of malnutrition (based on the Kenya Integrated Household Budget Survey 2005-2006), health expenditure (based on data from the Kenya National Bureau of Statistics) and population density. One county from each stratum was selected for sampling. In Uganda, 10 regions outlined by the 2011 Uganda Demographic and Health Survey were used to group districts and then two districts from each region were randomly selected for sampling. The second step for both countries involved sampling two to three facilities from each defined platform type within each county/region, including all national/provincial/referral hospitals and training institutions. ${ }^{12} 13$

The health facility surveys included six modules to cover each facility's finances, management, medical consumables, equipment, services, drugs and outputs. This information was supplemented by district level data (eg, salary and capital expenditure data) when available. Surveys evaluated facility inventories, healthcare delivery capacity and a variety of financial measures across different platforms. Detailed survey methods have been previously reported. ${ }^{12-14}$ Only selected pharmaceutical stock was evaluated based on necessity for treating prevalent communicable and non-communicable diseases. The ABCE study was reviewed and approved by Institutional Review Boards in Uganda (Ministry of Health, Makerere University, Uganda National Council for Science and Technology), Kenya (Africa Action Help-International, Ministry of Medical Services, Ministry of Public Health and Sanitation) and the University of Washington (Seattle, Washington, USA).

\section{Adult HF guidelines in Kenya and Uganda}

Essential medications listed for HF includes digoxin, enalapril, furosemide and hydrochlorothiazide in Kenya and only digoxin in Uganda. ${ }^{15} 16$ The Kenyan national clinical guidelines list only furosemide as first-line therapy, while ACE inhibitors are reserved for unresponsive patients and digoxin for patients with atrial fibrillation. ${ }^{17}$ The Ugandan national clinical guidelines for managing HF recommend first-line therapy with furosemide and ACE inhibitors. Some $\beta$-blockers (propranolol and atenolol), ACE inhibitors (lisinopril and captopril) and diuretics (furosemide) are listed as essential medications in one or both of these countries but for indications other than HF. ${ }^{15}{ }^{16}$ While variation exists in how medications are dispensed among facilities in each country, public hospitals should carry essential medications and dispense them free of charge.

\section{Data analysis}

Acute $\mathrm{HF}$ is a clinical diagnosis based on patient history and physical examination. We considered resources for the care of acute HF across two domains: diagnostic modalities and medication availability. For each diagnostic modality (ECG, radiography and ultrasound), we assessed whether a facility would be able to successfully acquire each study type based on the availability of at least one functional device and the availability of appropriate staff for acquisition. The ABCE survey did not specifically assess the availability of physicians or other providers trained in interpreting the results of these studies.

The ABCE survey did not ask about the treatment of HF directly or the availability of the full range of medications used to treat HF, most notably digoxin and enalapril. However, it did include two classes of medications used for treating acute HF: a loop diuretic (furosemide) and two ACE inhibitors (captopril and lisinopril), which will be referred to as 'selected ACE inhibitors.' Though not indicated for HF with reduced ejection fraction, we have included the two $\beta$-blockers included in the survey (atenolol and propranolol), which will be referred to as 'selected $\beta$-blockers', because of their use in managing hypertension and atrial fibrillation, which are common causes of HF. In addition to assessing the availability of a given combination of medications, we examined the prevalence of minor ( 1 day) and major $(8+$ days) stock-outs in the prior quarter (preceding 3 months).

We excluded dispensaries, community pharmacies, and counselling centres, as these facilities are not expected to provide acute medical care. Baseline facility data were described by platform type (ie, hospital, health centre, etc), ownership (public vs private), inpatient care availability and location (urban, semiurban, rural). Hospital data (beds, staff, medical providers) were described by both means and medians to account for positive skewness in facility size. Facility diagnostic and treatment capacity were analysed by country and platform type. The data were expressed using means, medians and percentages, where appropriate. All analyses were conducted using STATA statistical software, V.13.1.

\section{RESULTS}

\section{Facility profile}

The survey sampled a total of 340 facilities that provided direct patient care, 143 in Kenya and 197 in Uganda (table 1). Approximately two-thirds of the facilities in each country provided some level of inpatient care. The proportion of national, district and private hospitals in each country was similar. Less than one-third of the facilities in each country were located in an urban setting while rural facilities were more common in Uganda than Kenya. More than half of the facilities in both countries had public ownership and approximately one-half of the facilities in each country were managed at least in part by the Ministry of Health.

\section{Diagnostic imaging availability}

All of the facilities replied to the survey questions regarding the imaging modalities, radiography and ultrasound (table 2) for a $100 \%$ response rate. Less than $10 \%$ of the ambulatory facilities reported having functional and staffed radiography and ultrasound. Among hospitals, the reported rates for radiography and ultrasound were approximately $55 \%$ and $38 \%$ in Kenya and $36 \%$ and $46 \%$ in Uganda, respectively.

\section{Electrocardiography availability}

Only 101 facilities (47 in Kenya and 54 in Uganda) replied to survey questions regarding ECG, a response rate of $29 \%$. The 
Table 1 Country-specific facility data

\begin{tabular}{|c|c|c|c|c|c|}
\hline & Kenya $(n=143)$ & & Uganda $(n=197)$ & & All $(n=340)$ \\
\hline \multicolumn{6}{|l|}{ Hospitals } \\
\hline National and provincial/regional & \multicolumn{2}{|l|}{$11(7.7 \%)$} & \multicolumn{2}{|l|}{$14(7.1 \%)$} & $25(7.4 \%)$ \\
\hline District & \multicolumn{2}{|l|}{$18(12.6 \%)$} & \multicolumn{2}{|l|}{$11(5.6 \%)$} & $29(8.5 \%)$ \\
\hline Private & \multicolumn{2}{|l|}{$17(11.9 \%)$} & \multicolumn{2}{|l|}{$35(17.8 \%)$} & $52(15.3 \%)$ \\
\hline Subdistrict/Health centre IV & \multicolumn{2}{|l|}{$9(6.3 \%)$} & \multicolumn{2}{|l|}{$36(18.3 \%)$} & $45(13.2 \%)$ \\
\hline Total hospitals & \multicolumn{2}{|l|}{$55(38.5 \%)$} & \multicolumn{2}{|l|}{$96(48.7 \%)$} & $151(44.4 \%)$ \\
\hline \multicolumn{6}{|l|}{ Ambulatory } \\
\hline $\begin{array}{l}\text { Health centre and maternity (Kenya)/Health centre II } \\
\text { and III (Uganda) }\end{array}$ & \multicolumn{2}{|l|}{$71(49.7 \%)$} & \multicolumn{2}{|l|}{$91(46.2 \%)$} & $162(47.7 \%)$ \\
\hline Clinic & \multicolumn{2}{|l|}{$17(11.9 \%)$} & \multicolumn{2}{|l|}{$10(5.1 \%)$} & $27(7.9 \%)$ \\
\hline Total ambulatory & \multicolumn{2}{|l|}{$88(61.5 \%)$} & \multicolumn{2}{|l|}{$101(51.3 \%)$} & $189(55.6 \%)$ \\
\hline \multicolumn{6}{|l|}{ Location } \\
\hline Urban & \multicolumn{2}{|l|}{$43(30.1 \%)$} & \multicolumn{2}{|l|}{$54(27.4 \%)$} & $97(28.5 \%)$ \\
\hline Semirural & \multicolumn{2}{|l|}{$54(37.8 \%)$} & \multicolumn{2}{|l|}{$32(16.2 \%)$} & $86(25.3 \%)$ \\
\hline Rural & \multicolumn{2}{|l|}{$46(32.2 \%)$} & \multicolumn{2}{|l|}{$111(56.3 \%)$} & $157(46.2 \%)$ \\
\hline \multicolumn{6}{|l|}{ Facility type } \\
\hline Public & \multicolumn{2}{|l|}{$76(53.1 \%)$} & \multicolumn{2}{|l|}{$122(61.9 \%)$} & $198(58.2 \%)$ \\
\hline Private & \multicolumn{2}{|l|}{$67(46.9 \%)$} & \multicolumn{2}{|l|}{$75(38.1 \%)$} & $142(41.8 \%)$ \\
\hline \multicolumn{6}{|l|}{ Managed by Ministry of Health } \\
\hline Yes & \multicolumn{2}{|l|}{$76(52.8 \%)$} & $95(48.2 \%)$ & & $171(50.3 \%)$ \\
\hline No & $67(47.2 \%)$ & & $102(51.8 \%)$ & & $169(49.7 \%)$ \\
\hline Inpatient care available & & & & & \\
\hline Yes & $102(71.3 \%)$ & & $122(61.9 \%)$ & & $224(65.9 \%)$ \\
\hline No & $41(28.7 \%)$ & & $75(38.1 \%)$ & & $116(34.1 \%)$ \\
\hline Hospital data: & mean & median & mean & median & mean \\
\hline Facility beds & 163 & 38 & 115 & 34.5 & 135 \\
\hline Total staff at a facility & 280 & 61 & 116 & 38 & 175 \\
\hline Total doctors at a facility & 14.1 & 2 & 7.9 & 2 & 10.2 \\
\hline Total medical officers at a facility & 13.7 & 6 & 7.0 & 3 & 9.4 \\
\hline
\end{tabular}

majority of the respondent organisations were hospital-level facilities. Less than half of the hospitals in both countries and only two ambulatory facilities (both in Kenya) reported having functional and staffed ECG.

\section{Medication availability}

All of the facilities replied to the survey questions regarding medication availability for a $100 \%$ response rate (table 3 ). Among hospital-level facilities, the availability of furosemide and a selected $\beta$-blocker was over $90 \%$ in respondent facilities within each country. The availability of selected ACE inhibitors and all three medications was approximately one-half of the hospitals in Kenya and three-fourths of the hospitals in Uganda. Ambulatory facilities had lower rates of availability of all selected

Table 2 Diagnostic equipment availability

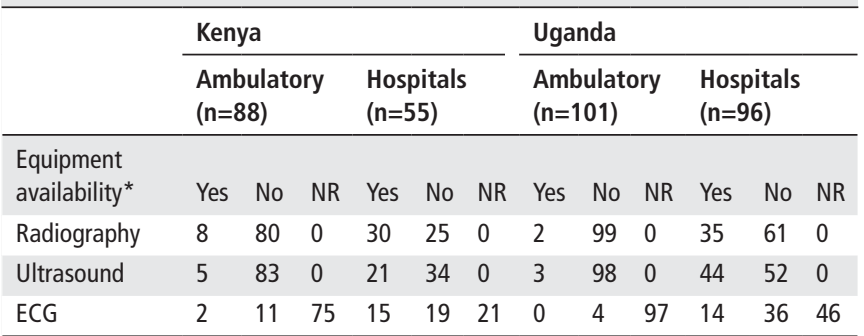

*Availability refers to a facility responding 'yes' to both having at least one functional machine and the requisite staff for acquisition. NR, No response provided to this set of survey questions. medications. Only 23\% of those facilities in Kenya and 22\% in Uganda had all three medications available. Availability of the selected ACE inhibitors in ambulatory care settings was particularly low in both countries.

\section{Medication stock-outs}

All of the facilities responded to the survey questions regarding minor and major stock-outs of medications on their formularies in the prior quarter (preceding 3 months) (table 4), Almost one-third of the hospitals in each country had a minor stock-out of at least one of the medication classes. These rates were even higher for ambulatory facilities. As expected, the rate of major

Table 3 Medication availability

\begin{tabular}{|c|c|c|c|c|c|c|c|c|}
\hline & \multicolumn{4}{|c|}{ Kenya } & \multicolumn{4}{|c|}{ Uganda } \\
\hline & \multicolumn{2}{|c|}{$\begin{array}{l}\text { Ambulatory } \\
(\mathrm{n}=88)\end{array}$} & \multicolumn{2}{|c|}{$\begin{array}{l}\text { Hospitals } \\
(\mathrm{n}=55)\end{array}$} & \multicolumn{2}{|c|}{$\begin{array}{l}\text { Ambulatory } \\
(n=101)\end{array}$} & \multicolumn{2}{|c|}{$\begin{array}{l}\text { Hospitals } \\
(n=96)\end{array}$} \\
\hline & Yes & $\%$ & Yes & $\%$ & Yes & $\%$ & Yes & $\%$ \\
\hline$\beta$-Blocker & 47 & 53 & 54 & 98 & 67 & 66 & 88 & 92 \\
\hline Atenolol & 34 & 39 & 52 & 95 & 38 & 38 & 77 & 80 \\
\hline Propranolol & 36 & 41 & 30 & 55 & 62 & 61 & 87 & 91 \\
\hline ACE inhibitor & 27 & 31 & 28 & 51 & 24 & 24 & 76 & 79 \\
\hline Captopril & 20 & 23 & 24 & 44 & 22 & 22 & 75 & 78 \\
\hline Lisinopril & 11 & 13 & 15 & 27 & 16 & 16 & 36 & 38 \\
\hline Furosemide & 50 & 57 & 54 & 98 & 73 & 72 & 90 & 94 \\
\hline All three medications & 20 & 23 & 27 & 49 & 22 & 22 & 74 & 77 \\
\hline
\end{tabular}

Carlson S, et al. Heart 2017;103:1874-1879. doi:10.1136/heartjnl-2016-310913 
Table 4 Medication stock-outs among facilities with availability

\begin{tabular}{|c|c|c|c|c|c|c|c|c|c|c|c|c|}
\hline & \multicolumn{6}{|c|}{ Kenya } & \multicolumn{6}{|c|}{ Uganda } \\
\hline & \multicolumn{3}{|c|}{ Ambulatory } & \multicolumn{3}{|c|}{ Hospitals } & \multicolumn{3}{|c|}{ Ambulatory } & \multicolumn{3}{|c|}{ Hospitals } \\
\hline & $\mathrm{n}$ & Minor & Major & $\mathrm{n}$ & Minor & Major & $\mathrm{n}$ & Minor & Major & $\mathrm{n}$ & Minor & Major \\
\hline$\beta$-Blocker & 47 & $10(21 \%)$ & $7(15 \%)$ & 54 & $2(4 \%)$ & $0(0 \%)$ & 67 & $7(10 \%)$ & $4(6 \%)$ & 88 & $8(9 \%)$ & $3(3 \%)$ \\
\hline Atenolol & 34 & $7(21 \%)$ & $5(15 \%)$ & 52 & $5(10 \%)$ & $3(6 \%)$ & 38 & $14(37 \%)$ & $6(16 \%)$ & 77 & $22(29 \%)$ & $9(12 \%)$ \\
\hline Propranolol & 36 & $9(25 \%)$ & $6(17 \%)$ & 30 & $1(3 \%)$ & $1(3 \%)$ & 62 & $9(15 \%)$ & $4(6 \%)$ & 87 & $7(8 \%)$ & $2(2 \%)$ \\
\hline ACE inhibitor & 27 & $7(26 \%)$ & $5(19 \%)$ & 28 & $7(25 \%)$ & $6(21 \%)$ & 24 & $9(38 \%)$ & $5(21 \%)$ & 76 & $10(13 \%)$ & $7(9 \%)$ \\
\hline Captopril & 20 & $6(30 \%)$ & $4(20 \%)$ & 24 & $8(33 \%)$ & $7(29 \%)$ & 22 & $9(41 \%)$ & $5(23 \%)$ & 75 & $16(21 \%)$ & $8(11 \%)$ \\
\hline Lisinopril & 11 & $1(9 \%)$ & $1(9 \%)$ & 15 & $5(33 \%)$ & $4(27 \%)$ & 16 & $2(13 \%)$ & $0(0 \%)$ & 36 & $8(22 \%)$ & $2(6 \%)$ \\
\hline Furosemide & 50 & $8(16 \%)$ & $6(12 \%)$ & 54 & $2(4 \%)$ & $1(2 \%)$ & 73 & $13(18 \%)$ & $4(5 \%)$ & 90 & $8(9 \%)$ & $5(6 \%)$ \\
\hline Any medication & 20 & $6(30 \%)$ & $4(20 \%)$ & 27 & $9(33 \%)$ & $6(22 \%)$ & 22 & $10(45 \%)$ & $6(27 \%)$ & 74 & $18(24 \%)$ & $10(14 \%)$ \\
\hline
\end{tabular}

Minor=1 day stock-out in the last quarter; major $=8+$ day stock-out in the last quarter.

stock-outs was lower in both countries. ACE inhibitors were the most commonly stocked out medication class in both countries.

\section{DISCUSSION}

Our study revealed that a large number of the facilities in Kenya and Uganda lack essential tools for diagnosing and treating HF (figure 2). Moreover, we found that selected ACE inhibitors were present in only half of Kenyan hospitals and three-fourths of Ugandan hospitals. Major stock-outs were noted in 22\% of Kenyan and 14\% of Ugandan hospitals. Our findings call for increased efforts in Kenya and Uganda to ensure the availability of drugs and equipment. Furthermore, while beyond the scope of this survey, it is crucial to provide training or have trained medical doctors to properly diagnose and treat HF.

The WHO does not provide specific recommendations regarding diagnostics for $\mathrm{HF}$ in resource-limited settings. It has been reported that screening patients for HF with a basic natriuretic peptide (BNP) followed by echocardiography (for abnormal BNP results) would be a cost-effective approach. ${ }^{18}$

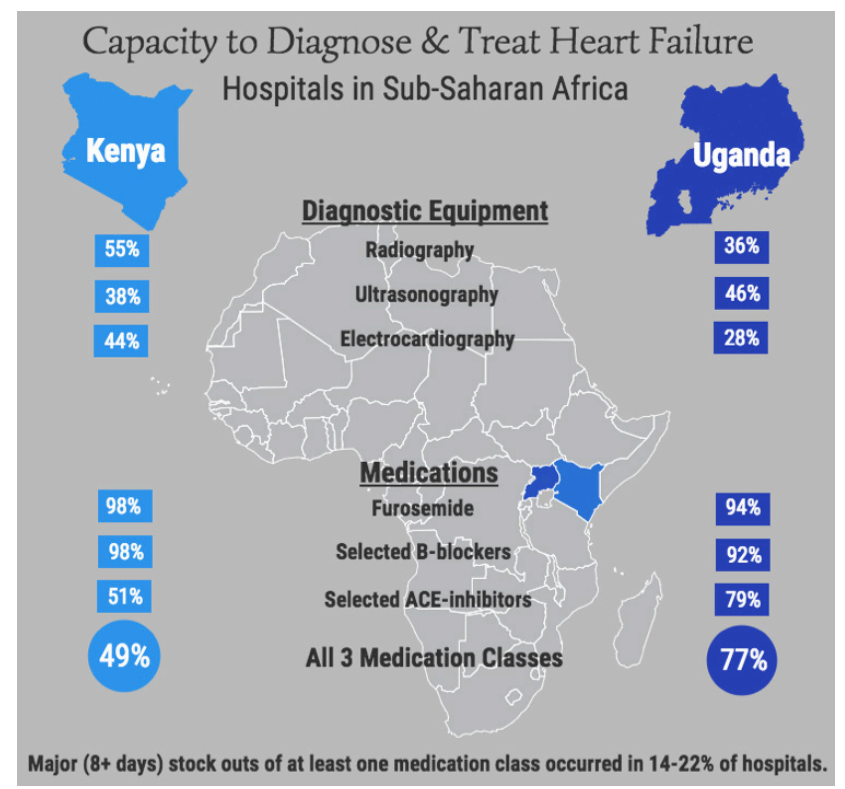

Figure 2 Capacity to diagnose and treat heart failure in hospitals in sub-Saharan Africa hospitals in Kenya and Uganda had good availability of furosemide and selected $\beta$-blockers but poor availability of selected ACE inhibitors. Major medication stock-outs in the preceding quarter (3 months) were fairly common. There was overall poor availability of essential equipment to diagnose heart failure.
However, the Kenyan and Ugandan clinical guidelines include hospital-based chest radiography, ECG and echocardiography as standard investigations for the diagnosis of HF but not laboratory BNP assessment. ${ }^{1719}$ Our analysis shows that only approximately one-third to one-half of hospitals (including subdistrict hospitals in Kenya and health centre IVs in Uganda) in each country had equipment and trained staff for all of these modalities. Notably, while we report on the availability of ultrasound, we were unable to assess whether it was used for cardiac imaging. A recent large meta-analysis of HF in LMIC included only one study (out of 42) that used echocardiographic criteria for diagnosis. ${ }^{10}$ The lack of echocardiographic utilisation is unfortunate, as training nurses and mid-level providers in simplified echocardiographic protocols has been recommended as an effective strategy to improve HF diagnosis and management in SSA. ${ }^{20}$

In 2010, the WHO outlined a core set of pharmacological interventions for non-communicable diseases in resource poor settings, including a cost-effective approach to congestive HF in district hospitals using ACE inhibitors, $\beta$-blockers and diuretics. This approach has an estimated cost of $\$ 150$ per disability-adjusted life year. ${ }^{21}$ Despite the cost-effectiveness, prescription rates of these medications for HF in SSA are suboptimal, with fewer than half of patients admitted to the hospital for HF receiving preventive medications. ${ }^{22}$ Specifically, one study from university hospitals in nine sub-Saharan countries (including Kenya and Uganda) reported prescription rates of $75 \%-85 \%$ for ACE inhibitors and less than 50\% for $\beta$-blockers at all time points. The same study reported prescription rates for loop diuretics to be only $15 \%$ at admission. ${ }^{22}$ Khatib et al recently estimated the availability of $\beta$-blockers, ACE inhibitors, aspirin and statins in ambulatory pharmacies in low-income countries to be between 3\% (rural) and 25\% (urban). ${ }^{23}$ Similarly, a 2008 WHO report showed that the mean availability of 15 essential first-line medications in public sector clinics was only $29.4 \%$ across eight African countries. ${ }^{24}$ However, to our knowledge, no study has previously reported availability of HF medications in hospitals in LMICs or in SSA. In our study, only one-half of hospitals in Kenya and three-fourths of hospitals in Uganda had one of the selected $\beta$-blockers, selected ACE inhibitors and furosemide available. More concerning is that a third of all hospitals surveyed reported at least one of these medication classes to never be available (supplementary appendix table 1).

Among facilities that carried the selected $\beta$-blockers, ACE inhibitors and furosemide on their formularies, medication stockouts in the prior quarter were common. A major stock-out of at least one of the medication classes was reported in 14\%-22\% of hospitals and $20 \%-27 \%$ of ambulatory facilities. While no 
study has previously reported stock-out rates for HF medications in SSA, stock-outs of essential medications for psychiatric disorders, communicable diseases and analgesia are common. Stock-out rates for these medications have been estimated to be between $20.5 \%$ and $45.5 \%$ or higher. ${ }^{25-27}$

Kenya and Uganda differ with respect to their national clinical guidelines and essential medication lists. ${ }^{15-17}{ }^{19}$ Notably, neither captopril nor lisinopril are listed as essential medications for any indication in Kenya, a difference which likely explains the lower availability of these medications in Kenya (51\%) relative to Uganda (79\%). Additionally, neither digoxin, the only HF medication listed as essential in both countries, nor enalapril, the only ACE inhibitor listed as essential in Kenya, was included in the ABCE survey. We, therefore, can make no inference regarding the availability of these medications.

Our findings show that poor access to essential health products in SSA extend to the care of HF. Decision making regarding national health expenditures is particularly complex in resource-limited settings. LMICs may appropriately forego investment in some areas to address more urgent national burdens. However, ACE inhibitors are among the most effective interventions for hypertension and therefore could contribute significantly to a health system capacity to prevent myocardial infarction, stroke and HF while simultaneously improving the capacity to treat HF when it does occur. Improving the availability of low-cost generic versions of these pharmaceuticals would directly address the rising burden of cardiovascular disease in these countries.

\section{LIMITATIONS}

For our analysis, we used existing data from a survey designed to evaluate healthcare across multiple conditions. There were low response rates to questions pertaining to ECG availability. This is secondary to an oversight in the survey design logic. Specifically, the survey logic included a gate question regarding whether the facility provided imaging services. If the answer to this question was 'no', the respondent was not prompted to answer specific questions about ultrasound, radiography and ECG. As such, it cannot be implied that the facilities that answered 'no' to the gate question would have answered 'no' to questions specific to ECG.

Additionally, pharmacy data were collected on a set of specific medications rather than investigating the complete set of pharmaceuticals available in a facility. It remains possible that other forms of ACE inhibitors and $\beta$-blockers that are neither included in the country's essential medication list nor asked about in the $\mathrm{ABCE}$ survey were available in the facilities. For instance, metoprolol and enalapril are commonly used medications in LMIC, but neither were included in the ABCE survey. Additionally, the questions in the survey asked about ultrasonography, but did not specifically assess echocardiography capabilities. Lastly, while the ABCE survey comprised a comprehensive set of public facilities, it did not include a comprehensive set of private facilities. Thus, we were limited in comparing public to private facilities and were able to capture the full capabilities of private facilities.

\section{CONCLUSION}

Our study shows that access to diagnostic tools and some medicines needed to diagnose and treat HF in Kenya and Uganda is limited. There is an urgent need to improve access to basic cardiovascular medications such as ACE inhibitors that can be used to treat hypertension in addition to HF with reduced ejection fraction. Low-cost generic versions of these medications

\section{Key messages}

What is already known on this subject?

The WHO has identified the use of ACE inhibitors, $\beta$-blockers and loop diuretics in hospitals as a cost-effective policy for treatment of acute heart failure (HF). However, no prior studies have examined the availability of standard diagnostic equipment or HF medications in hospitals in sub-Saharan Africa.

\section{What might this study add?}

This study is the first nationally representative assessment of the capacity to provide HF care in two countries within sub-Saharan Africa (SSA). Equipment and trained staffing for electrocardiography, ultrasonography and radiography were available in $44 \%, 38 \%$ and $55 \%$ of Kenyan hospitals and in $28 \%$, $46 \%$ and $36 \%$ of Ugandan hospitals, respectively. Additionally, only $49 \%$ of Kenyan hospitals and $77 \%$ of Ugandan hospitals had a medication from each class (selected $\beta$-blockers, selected ACE inhibitors and a loop diuretic) and $14 \%-22 \%$ of hospitals reported a major medication stock-out.

\section{How might this impact on clinical practice?}

Our study shows that essential HF medications and standard diagnostic equipment remain unavailable within many hospitals in SSA. Novel low-cost strategies need to be developed and tested in resource-limited settings to reduce these disparities in care.

need to be made broadly available to improve cardiovascular care in Kenya and Uganda.

Contributors All authors contributed to the manuscript as follows: SC, JA, GI: conception, design, data collection. SC, AW, GAR: analysis and interpretation of data. SC: drafting the article. HCD, GAR, GB, AHM, AS: critical revision for important intellectual content. SC, GAR, HCD: final approval of the version to be published. All authors read and approved the final manuscript.

\section{Competing interests None declared.}

Provenance and peer review Not commissioned; externally peer reviewed.

Data sharing statement The ABCE study data are available for free download at the Institute for Health Metrics and Evaluations Global Health Data Exchange website (http://ghdx.healthdata.org/series/access-bottlenecks-costs-and-equity-abceproject).

(c) Article author(s) (or their employer(s) unless otherwise stated in the text of the article) 2017. All rights reserved. No commercial use is permitted unless otherwise expressly granted.

\section{REFERENCES}

1 Amoah AG, Kallen C. Aetiology of heart failure as seen from a national cardiac referral centre in Africa. Cardiology 2000;93:11-18.

2 Bloomfield GS, Barasa FA, Doll JA, et al. Heart failure in sub-Saharan Africa. Curr Cardiol Rev 2013;9:157-73.

3 Damasceno A, Cotter G, Dzudie A, et al. Heart failure in sub-Saharan Africa: time for action. J Am Coll Cardiol 2007;50:1688-93.

4 Mendez GF, Cowie MR. The epidemiological features of heart failure in developing countries: a review of the literature. Int J Cardiol 2001;80:213-9.

5 Bukhman G. Heart failure in Africa: continuity or change? Heart Br Card Soc 2014; 100:1223-4

6 World Health Organization. Global action plan for the prevention and control of noncommunicable diseases 2013-2020. 2013. http://apps.who.int/iris/handle/10665/ 94384 (accessed 16 Jan 2016).

7 Ponikowski P, Anker SD, AlHabib KF, et al. Heart failure: preventing disease and death Worldwide. ESC Heart Fail 2014;1:4-25.

8 Gaziano TA. Economic burden and the cost-effectiveness of treatment of cardiovascular diseases in Africa. Heart 2008;94:140-4.

9 Jamison DT, Breman JG, Measham AR, et al. Disease control priorities in developing countries. 2nd ed. Oxford University Press; 645-662. 
10 Callender T, Woodward M, Roth G, et al. Heart failure care in low- and middle-income countries: a systematic review and meta-analysis. PLoS Med 2014;11:e1001699.

11 Dansereau E, Gakidou E, Ng M, et al. Trends and determinants of antiretroviral therapy patient monitoring practices in Kenya and Uganda. PLoS One 2015; 10:e0135653.

12 Health Service Provision in Kenya. Assessing facility capacity, costs of care, and patient perspectives. Seattle, WA: Institute for Health Metrics and Evaluation (IHME), 2014 http://www.healthdata.org/sites/default/files/files/policy_report/2014/ABCE/Kenya/ ABCE_Kenya_full_report_2014.pdf (accessed 18 Jan 2016).

13 Health Service Provision in Uganda. Assessing facility capacity, costs of care, and patient perspectives. Seattle, WA: Institute for Health Metrics and Evaluation (IHME). 2014. http://www.healthdata.org/sites/default/files/files/policy_report/2015/ABCE_ Uganda_finalreport_Jan2015.pdf (accessed 18 Jan 2016).

14 IHME. Access, bottlenecks, costs, and equity: ABCE project cross-country protocol. Seattle, WA: IHME: Institute for Health Metrics and Evaluation. 2015.

15 Ministry of Health. Essential medicines and health supplies list for Uganda (EMHSLU). Uganda: Ministry of Health; 2012 https://www.medbox.org/ug-drugs-medequipment/essential-medicines-and-health-supplies-list-for-uganda-emhslu-2012/ preview

16 World Health Organisation. Kenya essential medicines list 2010 (KEML). Republic of Kenya: Ministry of Medical \& Public Health. 2010. http://apps.who.int/medicinedocs/ documents/s18694en/s18694en.pdf

17 World Health Organisation. Clinical guidelines for management and referral of common conditions at levels 4-6: hospitals. In: Clinical management and referral guidelines. Republic of Kenya: Ministry of Medical \& Public Health; 2009:31-7. http:// apps.who.int/medicinedocs/documents/s21000en/s21000en.pdf

18 Heidenreich PA, Gubens MA, Fonarow GC, et al. Cost-effectiveness of screening with B-type natriuretic peptide to identify patients with reduced left ventricular ejection fraction. J Am Coll Cardiol 2004;43:1019-26.
19 World Health Organisation. Uganda clinical guidelines 2012 - national guidelines for management of common conditions. Uganda: Ministry of Health; 2012. http://apps. who.int/medicinedocs/documents/s21741en/s21741en.pdf

20 Kwan GF, Bukhman AK, Miller AC, et al. A simplified echocardiographic strategy for heart failure diagnosis and management within an integrated noncommunicable disease clinic at district hospital level for sub-Saharan Africa. JACC Heart Fail 2013;1:230-6

21 World Health Organisation. Package of essential NCD interventions for primary health care: cancer, diabetes, heart disease and stroke, chronic respiratory disease. http:/ www.who.int/cardiovascular_diseases/publications/pen2010/en/ (accessed 5 Jan 2016).

22 Damasceno A, Mayosi BM, Sani M, et al. The causes, treatment, and outcome of acute heart failure in 1006 Africans from 9 countries. Arch Intern Med 2012;172:1386-94.

23 Khatib R, McKee M, Shannon $\mathrm{H}$, et al. Availability and affordability of cardiovascular disease medicines and their effect on use in high-income, middleincome, and low-income countries: an analysis of the PURE study data. Lancet 2016;387:61-9.

24 Cameron A, Ewen M, Ross-Degnan D, et al. Medicine prices, availability, and affordability in 36 developing and middle-income countries: a secondary analysis. Lancet 2009;373:240-9.

25 Wagenaar BH, Gimbel S, Hoek R, et al. Stock-outs of essential health products in Mozambique - longitudinal analyses from 2011 to 2013. Trop Med Int Health 2014;19:791-801.

26 Wagenaar BH, Stergachis A, Rao D, et al. The availability of essential medicines for mental healthcare in Sofala, Mozambique. Glob Health Action 2015;8:27942.

27 Harding R, Simms V, Penfold $S$, et al. Availability of essential drugs for managing HIV-related pain and symptoms within 120 PEPFAR-funded health facilities in East Africa: a cross-sectional survey with onsite verification. Palliat Med 2014;28:293-301. 